\title{
ARTÍ́CULOS
}

\section{Interculturalidad en contexto mapuche 1}

\section{Interculturality in mapuche context}

\author{
Recepción: Diciembre - 2008 \\ Aprobación: Abril - 2009
}

Daniel Quilaqueo Rapimán *

Resumen

En este artículo se presentan dos aspectos generales que permiten explicar la dinámica intercultural. El primero se refiere a los significados de los términos pluricultural y multicultural y se señala que la dinámica de la génesis de lo intercultural (y lo cultural) se sustenta en lo epistemológico y en lo histórico. El segundo aspecto plantea la dificultad (epistemológica) entre 'contexto de descubrimiento' y 'contexto de justificación', para la reflexión de los contextos en los que se desarrollan las relaciones interculturales entre mapuches y no mapuches. Se complementa con la noción de inducción para la comprensión del problema epistemológico que plantea la revelación de los contextos interculturales. Se concluye que la dinámica de la génesis del concepto intercultural permite la posibilidad de pensar la interculturalidad para formarse y problematizar situaciones interculturales en investigación. La diversidad y la complejidad de representaciones (descripciones) de lo intercultural, en una época donde los cambios profesionales son frecuentes, obligan a los investigadores considerar su extensión, la diversidad y la profundidad de los problemas que presentan los contextos interculturales.

Palabras clave: multicultural, interculturalidad, contexto, dinámica intercultural

\begin{abstract}
This article presents two general aspects that allow to explain the intercultural dynamics. The first aspect refers to the meanings of pluricultural and multicultural concepts, showing that the dynamics of interculturality (and culturality) genesis is based on the epistemology and history. The second aspect establishes the difficulty (epistemological) between 'discovery context' and 'justification context' in order to reflect about contexts in which intercultural relationships between Mapuche and non Mapuche are developed. This is complemented by the induction notion to understand the epistemological problem presented because of the intercultural contexts revealing. In conclusion, the dynamics of the intercultural concept genesis allows the possibility of thinking of interculturality in order to form oneself and discuss intercultural situations on research. The diversity and complexity of interculturality representations (descriptions) during times when professional changes are frequent, force researchers to consider their extension, diversity and depth of problems that intercultural contexts present.
\end{abstract}

Key words: multicultural, interculturality, context, intercultural dynamics.

\footnotetext{
* Escuela de Educación Media Científica, Facultad de Educación, Universidad Católica de Temuco. Correo: dquilaq@uctemuco.cl

1 Este artículo es parte de los resultados del proyecto Fondecyt $N^{\circ} 1085293$. Parte de éste fue presentado como conferencia en REUNA (Red Universitaria Nacional), en junio de 2008. Las conferencias fueron realizadas por el Centro de Investigación en Educación en Contexto Indígena e Intercultural del Núcleo Iniciativa Científica Milenio (CIECII) P075-039-F, de la Universidad Católica de Temuco.
} 


\section{Introducción}

La interculturalidad es fundamentalmente una propuesta de convivencia entre grupos y personas que comparten saberes y conocimientos culturales con el fin de tener un desarrollo social, cultural, económico y político común en un determinado país. En este artículo se presentan algunas ideas para elaborar un marco teórico que trata de dar respuesta a la interrogante de cómo comprender la interculturalidad en las relaciones socioculturales entre los mapuches y los no mapuches en Chile. Para este propósito, se han considerado dos aspectos generales que permitirán explicar la dinámica intercultural. El primer aspecto se refiere a los significados de los términos pluricultural y multicultural, y a la noción de contexto. El segundo aspecto aborda algunos procesos en la dinámica de la génesis del concepto intercultural, la posibilidad de considerar la interculturalidad para la formación y la problematización de situaciones interculturales que están en investigación.

\section{Pluricultural, multicultural y contexto}

Los términos pluricultural y multicultural aunque son intercambiables, ambos deben ser considerados como referencias para investigar la interculturalidad en los contextos de vida de los mapuches (Quilaqueo; Quintriqueo, 2008). Pluricultural y multicultural, en este trabajo, califica la existencia de varias o de múltiples culturas presentes en un mismo territorio. Lo multicultural se refiere a las acciones de los Estados hacia los territorios y miembros de los pueblos indígenas. El territorio, para ambos casos puede comprender un lugar, una comunidad mapuche de una región determinada, un país, un continente, hasta el planeta entero. Las culturas que pueden representar estos términos están relativamente separadas por diversas razones y formas. Considerando el planeta, la separación de las grandes zonas culturales o de civilizaciones diferentes es de tipo geográfico e histórico (geohistórico). Esta multiculturalidad de civilización es evidente entre Europa, América y los otros continentes. Las grandes zonas culturales diferentes, particularmente las religiosas, han sido regiones aisladas unas de otras (Breton, 1987; Todorov, 1993; Wieviorka, 1996).
Según Demorgon (1998), las grandes orientaciones culturales históricas son producto de las respuestas que argumentan la salida del período societal precedente al de las sociedades comunitarias, por ejemplo, la forma societal de la realeza (en el caso de Europa) o imperial (en el caso de China), que durante mucho tiempo luchó con las sociedades comunitarias. De los choques y alianzas entre estas dos grandes orientaciones culturales históricas, surgieron sociedades singulares. Los acuerdos y disputas entre las monarquías para expandir su poderío con los actores económicos, contribuyeron a engendrar una tercera forma de sociedad y cultura. Esta nueva forma de organización, altamente diversificada entre ellas, es la de las naciones comerciantes. Sin embargo, según este mismo autor, a fines del siglo $X X$, se dan las condiciones que generan una cuarta forma de sociedad, cuya denominación es la sociedad de la información, que se extiende rápidamente a nivel mundial. Estos cuatro tipos de sociedades están, en los hechos, necesariamente presentes en lo multicultural del planeta. No obstante, este autor sostiene que "...en numerosas regiones del mundo se encuentran todavía sociedades comunitarias. Estas han subsistido por razones geográficas ligadas a condiciones biológicas extremas" (Demorgon (1998: 30; traducción propia). De esta manera, la noción de multiculturalidad traduce las realidades geográficas e históricas de los distintos pueblos hoy insertos en los diversos Estados pero, al mismo tiempo, es evidente que las principales formas culturales societales se desarrollaron a partir de los intercambios singulares con las sociedades comunitarias. Estos intercambios fueron producidos por relaciones generalmente violentas.

Esta ha sido la primera fase de transición entre dos grandes formas culturales societales. Durante este largo período los excesos de violencia y también de acuerdos, en los encuentros entre militares y sociedades comunitarias, y la importancia de los intercambios comerciales puso principalmente en evidencia la interculturalidad. Esto se demuestra en los encuentros y contactos entre los mapuches con comerciantes y misioneros, sean estos violentos o pacíficos (Coña, 1984; Bengoa, 1996). Por el contrario, el distanciamiento y el aislamiento entre las sociedades y grupos humanos pone en evidencia la situación multicultural 
(Wieviorka, 1996). Sin embargo, la interculturalidad, cuando ha asumido formas de estrategia y coyuntura de los grupos dominantes, ha producido nuevas situaciones de multiculturalidad (Hess, Wulf 1999). En efecto, más allá de los momentos de contacto y de experiencia religiosa, política, económica, por ejemplo, y más allá de las guerras desgarradoras, muchas veces, los aspectos de sobrevivencia buscada por las sociedades comunitarias, impusieron acuerdos que conllevaron a nuevas condiciones de separación en el territorio que era común a todos (Cantoni, 1978; Villalobos et al., 1982; Foerster y Montecino, 1988; Orellana, 1994; Faron, 1997). Este es el caso de la mayoría de los pueblos indígenas del continente americano, luego de la formación de las repúblicas independientes de España, Portugal e Inglaterra. Entonces, en la etapa de la colonización, el fenómeno de la conquista de los territorios ocupados por los indígenas generó la creación de zonas de refugio o de reducciones (Aguirre Beltrán, 1967). Allí el Estado estableció relaciones interétnicas con las comunidades indígenas, lo que originó una situación multicultural, ya que se impuso una relación monocultural impulsada por la escolarización, donde la cultura y, particularmente, los saberes educativos indígenas no fueron considerados por los no indígenas, lo cual dificultó la interculturalidad entre indígenas y no indígenas (Quilaqueo, 2005).

Con el tiempo, el enfoque multicultural comienza a cambiar debido a la evolución demográfica de los pueblos indígenas. Esta situación ha posibilitado numerosas proximidades espaciales y de entornos culturales parcialmente comunes, por ejemplo, la cohabitación entre pueblos indígenas y no indígenas en una misma zona geográfica, de una misma cultura singular global de un país (es el caso de Chile) y sus actividades generales como la política y el comercio. Sin embargo, esta cohabitación ha sido establecida tanto desde el aspecto jurídico por el Estado como por la realidad de costumbres diferentes, sin poseer el conocimiento cultural de los pueblos indígenas (Cantoni, 1973). En síntesis, esto ilustra, de alguna manera, la percepción de la 'sociedad multicultural' y del 'derecho de las minorías'; esto se observa en la voluntad de promover el trato con las poblaciones discriminadas y las más desfavorecidas. Es decir, es lo que se observa en la agenda política y en las leyes de 'discriminación positiva' o de la 'acción afirmativa' de los Estados (Costa-Lascoux, 1999). Las culturas particulares de los países son sistemas unidos a las instancias de sus vivencias culturales estratégicas. Por ejemplo, en el caso de Estados Unidos, la toma de conciencia de la cuestión multicultural data del año 1981 (Glazer, 1996). Aunque por primera vez se utiliza el término multiculturalismo en los artículos de la prensa norteamericana, su expansión es rápida, ya que diez años más tarde, en 1992, el mismo autor acusa, para la misma muestra, 2.000 recurrencias del término.

En suma, si lo multicultural significa una cierta coexistencia de yuxtaposición de las culturas y si lo intercultural significa momentos de contactos violentos o pacíficos, tendríamos dos nociones claramente separadas, y las dos necesarias, Lo que de alguna manera, atenuaría la oposición entre las dos orientaciones. En efecto, cada una de ellas es en realidad constituida de pertinencia en su zona cultural. Así, el multiculturalismo se presenta bajo dos formas: la de yuxtaposición y la de segregación. Para la yuxtaposición, vemos el caso de los británicos en India: la cultura británica se yuxtapone a las culturas, muy diversas, de este país. Aquí, es el conquistador quien impone la paz al país obligando a respetar las culturas diferentes. Sin embargo, en algunos casos, es el conquistador quien tolera la cultura por medio de la explotación de las poblaciones marginalizadas. En este caso, se observa un multiculturalismo de segregación y no de yuxtaposición pacífica o de tolerancia. Este ha sido el caso de África del Sur, donde se ha experimentado una conversión política hacia una coexistencia menos desigual y más pacífica (Demorgon, 2000). De esta manera, se puede concluir que la multiculturalidad presenta una imagen ambigua.

Se observa que cuando el multiculturalismo ha prevalecido, primero fue por imposición de su presencia a los "otros" que no lo deseaban. Una vez triunfante, esta imposición no siempre fue exterminadora, encuentra ventajas para coexistir con los pueblos indígenas. Es lo que ocurrió con las culturas comunitarias de grupos indígenas de Canadá, por ejemplo, donde "...el multiculturalismo ha llegado a ser exterminador, segregacionista y, después, 
se ha mostrado indiferente, pero también ha logrado una posición moral que busca promover el respeto de las diferencias" (Demorgon, 1989: 33, traducción propia). Estos aspectos se presentan también en la mayoría de los pueblos indígenas de América Latina, desde un contexto político en el que ha primado una relación unilateral por parte de los Estados nacionales, principalmente, desde el indigenismo (Aguirre Beltrán, 1982; Barre, 1983).

Generalmente, en el contexto actual de los pueblos indígenas, las relaciones con los Estados nacionales son de tipo multicultural. Son pueblos económicamente pobres, con una gran riqueza cultural y de lenguas, donde las relaciones interculturales e interétnicas se apoyan principalmente en la pobreza material como símbolo del ser indígena y no en lo cultural. En el caso mapuche, las relaciones entre las comunidades y el Estado han sido relaciones interétnicas que, desde los años 80 del siglo pasado, incluyen los conceptos multicultural e intercultural para referirse a las relaciones establecidas en el proceso de democratización del país. En efecto, los mapuches constituyen uno de los pueblos indígenas más numerosos del país y han sido agrupados por el Estado en dos tipos de contextos socioculturales: 1) En reducciones o lotes de tierra, donde el Estado chileno reagrupó a los sobrevivientes de la 'Pacificación de la Araucanía'; 2) En nuevas comunidades como consecuencia de adjudicaciones de tierras de acuerdo con la Ley Indígena $\mathrm{N}^{\circ}$ 19.253, de Protección, Fomento, y Desarrollo de los Indígenas de Chile. Sin embargo, la mayor parte la población mapuche vive en el medio urbano como efecto de la sobrepoblación de sus comunidades de origen ${ }^{2}$.

Desde otro punto de vista, los conceptos multicultural e intercultural se relacionan, fundamentalmente, con la educación intercultural bilingüe, educación sugerida por dirigentes indígenas e intelectuales de las ciencias sociales para contextualizar los saberes escolares con los saberes culturales y educativos de los pueblos indígenas (Quilaqueo, Quintri- queo, Cárdenas, 2005). Para estos autores, el concepto intercultural se refiere a una relación que compromete saberes culturales y educativos en las relaciones intergrupales y entre personas. Sin embargo, en la perspectiva de las instituciones gubernamentales subyace el punto de vista monocultural donde lo intercultural es, principalmente, para los indígenas. En esta comprensión del concepto intercultural, aflora la idea del multiculturalismo practicado en países donde a las comunidades indígenas se las considera como comunidades culturales separadas (no integradas) en un país, mientras que para los autores Vignaux, Fall y Turgeon (1998), la interculturalidad se refiere al conjunto de procesos psíquicos, relacionales, grupales e institucionales generados por las interacciones de culturas en un tipo de relación de intercambios recíprocos, en la perspectiva del respeto de una identidad cultural de los grupos y personas involucradas.

La noción de contexto que se utiliza en este artículo se refiere a la distinción entre 'contexto de descubrimiento' y 'contexto de justificación'. La idea que subyace en las investigaciones llevadas a cabo en el medio mapuche es construir una epistemología de base para la reflexión de los contextos en los que se desarrollan las relaciones interculturales entre mapuches y no mapuches. De acuerdo con las definiciones aportadas por Muñoz y Velarde (2000), el 'contexto de descubrimiento' es todo aquello que tiene que ver directamente con la actividad científica orientada a descubrir y el 'contexto de justificación' es el conjunto de actividades destinadas a la prueba, comprobación o verificación de la validez de lo descubierto. Otra noción que complementa la comprensión del problema epistemológico que plantea el develamiento de los contextos interculturales es la noción de inducción. La inducción, para este propósito, se refiere a una concepción que permite justificar un descubrimiento mediante un trabajo empírico de inducción confirmatoria, puesto que se puede utilizar más en el 'contexto de justificación' que en el 'contexto de descubrimiento'. Esto

\footnotetext{
${ }^{1}$ La reducción se refiere al tipo de tenencia de tierra que el Estado chileno creó para reagrupar a los mapuches sobrevivientes de la guerra denominada "Pacificación de la Araucanía". Hoy día, una parte de la población mapuche habita en contextos rurales y ocupa lotes de tierra en nuevas comunidades, como resultado de la organización sociopolítica creada por el Estado (Ley Indígena $\mathrm{N}^{\circ}$ 19.253). Sin embargo, la mayoría habita en contextos urbanos.
} 
permite una justificación de las conexiones que implica este tipo de conocimiento, para dar cuenta de los procesos científicos que permitan comprender los tipos de relación entre las comunidades indígenas y las no indígenas. Sin embargo, la inducción se usaría más bien para justificar una teoría en función de los datos de las observaciones que para encontrarla (Reichenbach, 1953).

\section{Dinámica y génesis de la interculturalidad}

La interculturalidad es parte de la dinámica de las sociedades que han elegido procedimientos de unión cultural, primero al interior de ellas mismas y, enseguida, en su relación con las otras culturas. Se parte de la idea que cada cultura posee características que son trascendentales y relevantes también para las otras culturas. Desde esta perspectiva, mantienen siempre al mismo tiempo tanto lo auténtico como la atracción por la otra cultura. Sin embargo, esta interculturalidad, en su dinámica y génesis, sustenta la idea de que las otras culturas se acomodan más bien de manera voluntaria debido a ciertos intereses (Demorgon, 2000). El conocimiento del "otro" es la base de lo que se califica como lo intercultural, son conocimientos estratégicos que permiten representar intenciones del pasado o del presente. Esto refleja bien actitudes culturales que se les atribuyen a los países coloniales con culturas diferentes. Para AbdallahPretceille y Porcher (1999), lo intercultural se inscribe en los saberes y las prácticas de todas las sociedades que las irrigan, mezclando las discontinuidades y las apropiaciones, avanzando como el agua, pero siendo durante mucho tiempo disimuladas en las sociedades contemporáneas.

De esta manera, la dinámica en la génesis de lo intercultural (y lo cultural) se sustenta en dos aspectos principales: en lo epistemológico y en lo histórico. Según Demorgon (2000), estos aspectos han permitido verificar la realidad de, por lo menos, cuatro grandes tipos de antecedentes necesarios para el estudio de la dinámica cultural e intercultural. Primero, las culturas y lo intercultural deben definirse teniendo en cuenta las instituciones sociales. Pero se debe tener en cuenta que la institución social dominante como la economía, o la religión o la política por ejemplo, no definen por sí misma la génesis cultural; es necesario considerar otros aspectos como la historia propia de los pueblos indígenas y los conocimientos culturales entre otros. Puesto que, es el caso de los pueblos indígenas que continúan manifestando y movilizando sus propias fuerzas, mediante estrategias diversas de muchos actores. Segundo, las culturas y lo intercultural se vinculan siempre con un proceso histórico en curso. Tercero, el futuro de las culturas y lo intercultural dependen necesariamente de las orientaciones y de las estrategias de todos los actores. Y cuarto, los tres aspectos anteriores permiten plantear que existe un sector social dinámico nuevo que se ocupa de los otros sectores. Las estrategias de los actores se van a determinar en relación a esta dinámica actual, pero van a hacerla también a partir de las grandes culturas societales históricas.

En resumen, la cultura no es un sector yuxtapuesto a otros, ella atañe a todos los sectores. No existe un sector cultural separado, aislado y yuxtapuesto. La dimensión cultural se extiende a cada sector y a su totalidad social y societal, es decir, un estado específico de todo sector de la actividad humana. Lo intercultural es intersectorial y las culturas y lo intercultural son tanto sectoriales como intersectoriales.

\section{Las culturas y lo intercultural}

Las culturas y lo intercultural conjugan, de hecho, pasado, presente y futuro. Desde esta perspectiva, se observa el desarrollo de lo intercultural haciéndose énfasis en dos tentativas de explicación. Una es la perspectiva culturalista, donde la cultura se considera como el aspecto determinante. Y la segunda perspectiva sería la actualista, donde se considera que solo la estrategia actual es pertinente (Demorgon, 2000). Ahora bien, si estos dos enfoques fueran reconocidos, habría posibilidades para hacer las preguntas interculturales como un conjunto complejo de interacción, cuyo acontecer no se puede tratar fácilmente, puesto que las culturas y lo intercultural son históricos. Por ejemplo, las consecuencias a que llevan las conductas económicas y de información ligadas a organizaciones e instituciones como los grandes bancos y bolsas nacionales: Banco Mundial, Fondo Monetario Internacional, etc., es decir, hoy día, la globalización en curso es la evolución 
del conjunto cultural mundial que se presenta también determinante. De ahí, la necesidad de tener una representación más exacta, pero a la vez más compleja, al considerar toda la temporalidad de pasado, presente y futuro de los pueblos indígenas.

En tanto, las culturas singulares nacionales están compuestas de complejas interacciones evolutivas locales dependientes de las grandes orientaciones culturales históricas. En este sentido, Demorgon (2000) ha establecido tres grandes categorías culturales: comunitarias, de los reinos e imperios y nacional-comerciante. Sin embargo, queda abierta la posibilidad de génesis de una nueva composición cultural-societal. Se observa que las grandes culturas societales históricas no se generaron de una sola vez y se puede constatar que son el resultado de saberes y conocimientos construidos que, en efecto, han presentado formas muy diversas. Se observa también que la crisis que se presenta actualmente no es solo sectorial-económica, sino también cultural.

\section{Pensar la interculturalidad}

Pensar la interculturalidad es afirmar que es posible acceder a formarse en los saberes y conocimientos y a formarse en la práctica de su propia cultura, pero al mismo tiempo en otra cultura, es decir, en la cultura del 'otro' con quien compartimos en un mismo país espacios sociales y territorio. Actualmente, el 'otro' se ha generalizado, pero también se ha acercado. Él viene a nosotros o nosotros vamos a él a través de los desplazamientos, de la comunicación a distancia y existen más posibilidades de encuentros (Quilaqueo, 2005). Formarse para los encuentros, para los intercambios, para la cooperación y para enfrentar los conflictos hoy día es una tarea que concierne cada vez a más personas. Sin embargo, se debe estar consciente de la diversidad y de la complejidad de los intercambios interculturales para no desarrollar una formación superficial que sea fácilmente sobrepasada por la realidad actual y futura.

Se puede pensar también que lo intercultural representa más bien un efecto de moda o una trampa. Desde la perspectiva de las comunidades mapuches, al igual que la de muchos pueblos indígenas de América Latina, el 'otro' o los 'otros', a partir de su ocupación histórica del territorio donde estaban establecidas las comunidades mapuches, son los colonos españoles quienes dominaron desde su lógica cultural a la población mapuche (Todorov, 1991). También, los 'otros' son los misioneros, los técnicos y especialistas (científicos) que crearon las universidades $\mathrm{y}$, en general, la educación. Además, actualmente, podemos enumerar otras instituciones sociales (educación, política, economía, religión, etc.), las ONG, etc., que continúan desarrollando tipos de relaciones desde una perspectiva histórica, sectorial o intersectorial.

Para pensar la interculturalidad, desde una perspectiva más filosófica, los conceptos de interculturalidad y cultura permiten comprender las dificultades que presenta la comprensión del concepto de interculturalidad. Para Fornet-Betancourt (2001), la interculturalidad no se refiere a la incorporación del 'otro en lo propio', como en lo religioso, moral o ético, sino que busca más bien la transfiguración de lo propio y de lo ajeno sobre la base de lo común y en vista a la creación de un espacio común determinado por la convivencia. Del mismo modo, el concepto de cultura debe comprenderse desde el problema teórico que presenta, puesto que convergen las ciencias humanas y la filosofía en su definición como grandes categorías del quehacer epistémico. Es un concepto que debe tener en cuenta el concepto de sociedad para sobrepasar la terminología provista por el 'positivismo' y 'cientificismo' (Salas, 2003).

Entonces, los contactos y relaciones interculturales, cualquiera sea el motivo y forma, son parte cada vez más frecuente en nuestra vida económica, educacional, política, religiosa y de las comunicaciones. En consecuencia, la formación a la interculturalidad se presenta como una dimensión que, obviamente, no puede estar ausente en la formación universitaria. Sin embargo, es posible constatar que en la mayoría de las universidades este aspecto no forma parte de los contenidos obligatorios. Es posible observar que cuando la interculturalidad se incluye como contenido en los programas se realiza mediante un enfoque descriptivo-comparativo, generalmente, con un acento en lo lingüístico, informaciones culturales prácticas o de civilización. Cierta- 
mente, esto es un aporte importante, pero es imprescindible reconocer que hay otras exigencias: identificar y categorizar la producción de saberes y crear nuevos métodos para formar. Es necesario reunir los datos sobre saberes y conocimientos indígenas, por ejemplo, dándolos a conocer de una manera crítica para formar a los no indígenas. En efecto, se debería abogar por un cambio mental que nos obligue a modificar profundamente nuestros viejos puntos de vistas.

Sin embargo, la cuestión intercultural es hoy todavía muy conflictiva. Para algunos, el término está mal elegido. Mejor sería hablar de multiculturalidad y de multiculturalismo. Para los partidarios de lo intercultural, el término se justifica en la medida que la globalización permita que las interacciones estén por sobre las yuxtaposiciones o las segregaciones, pero por otro lado, las opiniones también están divididas. Para algunos autores, lo intercultural se define como el ideal de mañana, pero, por otra parte, es considerado como el espacio para la alteración de las culturas de la cual no se desea renunciar (Gosselin y Ossebi, 1994; Demorgon, 2000), es decir, que se considera como negativo y destructor.

Entonces, ¿cómo podemos comprender las situaciones interculturales? Lo intercultural se puede definir en función de las situaciones sociales, pero particularmente profesionales, en las cuales se manifiesta. Estas son numerosas, pero las que se consideran más frecuentes son las de situaciones interpersonales e intergrupales, por ejemplo, las ocupaciones como negociador, diplomático, jurista, agente de desarrollo, psicólogo, informador de los medios de comunicación y, por supuesto, formador y educador. En síntesis, las situaciones interculturales se presentan en numerosos campos: la enseñanza, el trabajo social, la asesoría de empresas comerciales, o la dirección de empresas relacionadas con lo intercultural, la gestión política de una región o país, las relaciones benéficas, etc.

\section{Problematizar las situaciones interculturales}

Con el fin de estudiar las situaciones interculturales, se hace necesario plantear hipótesis, supuestos y preguntas, sin olvidar la gran matriz cultural que a su vez es inter- cultural, pero al mismo tiempo en función de las situaciones interpersonales e intergrupales en las cuales se presenta. El desarrollo muy distinto de las situaciones interculturales, interpersonales e intergrupales, casi siempre de carácter voluntario, no debe hacernos olvidar la gran matriz intercultural atada a las múltiples estrategias de las sociedades que se expresan mediante los sectores políticos, económicos, de información y religioso, entre otros. De esta manera, un enfoque intercultural histórico y estratégico es fundamental para toda comprensión más singular.

Asimismo, es necesario considerar que los investigadores del tema también son partícipes las situaciones interculturales. Sin embargo se debe ir más allá de las implicancias inmediatas, es decir, debe recurrirse a la problematización. Para ello, algunos ejemplos de temas de problematización permiten mostrar que el investigador también está implicado en la interculturalidad: entre mayoría y minoría en un a sociedad nacional; entre el emigrado y la comunidad de acogida; entre cultura dominante (cultura del Estado) y cultura dominada (por ej., cultura mapuche o cultura regional); entre la cultura regional, o mapuche y la cultura de la ecología; entre la cultura nacional o regional y la cultura virtual; entre la cultura de la escuela y la cultura de la comunidad mapuche, etc.

En conclusión, todos estos temas transversales constituyen 'metatemas', que dicen relación con lo intercultural desde la perspectiva del campo ocupacional; pero situar lo intercultural solamente desde la perspectiva de las ocupaciones lo transforma en un instrumento. De esta manera, sería despojado de su complejidad y las perspectivas de las prácticas profesionales se empobrecerían. En una época donde los cambios profesionales son frecuentes cometeríamos un error al no considerar su extensión, la diversidad y la profundidad de los problemas interculturales. Es cierto que cada uno lo limitará al área donde trabaja y al nivel social en el cual se sitúa, pero esta es la razón por la cual tenemos presente en esta exposición la diversidad y la complejidad de representaciones (descripciones) de lo intercultural, para proponer pasar, si es necesario, de un actor a otro, de un nivel a otro, en función tanto de la época como de su interés, de su actividad profesional y de su libertad. 


\section{BIBLIOGRAFÍA}

ABALLAH-PRETCEILLE, M., PORCHER, L. (1999). Diagonales de la communication interculturelle. Ed. Anthropos, Paris.

AGUIRRE BELTRÁN, G. (1967). Regiones de refugio. El desarrollo de la comunidad y el proceso dominical en mestizo América. Instituto Indigenista Interamericano, México.

AGUIRRE BELTRÁN, G. (1982). El proceso de aculturación. Ediciones de la Casa Chata, México.

BARRE, M. CH. (1983). Ideologías indigenistas y movimientos indios. Siglo Veintiuno Editores, México.

BENGOA, J. (1996). Historia del Pueblo Mapuche. $5^{a}$ Edición. Santiago: Ediciones Sur.

BRETON, R. (1987). Géographie des civilisations. Presses Universitaires de France, Que sais-je ? $N^{\circ} 2317$, Paris.

CANTONI, W. (1978). "Relaciones del mapuche con la sociedad nacional chilena". En Un estudio sobre las relaciones entre los grupos étnicos en el Caribe de lengua inglesa, Bolivia, Chile y México. UNESCO: Paris.

COÑA, P. (1984). Testimonio de un cacique mapuche. Primera edición Imprenta Cervantes, 1930, segunda edición ICIRA, 1973. Pehuén Editores, 1984, Santiago.

COSTA-LASCOUX, J. (1999). Citoyenneté et multiculturalisme. En Hilly, M. et Lefebvre, M. (Sous la direction), Identité collective et altérité. L'Harmattan, Paris, France pp. 53-76

DEMORGON, J. (1998). L'Histoire Interculturelle des Sociétés. Anthropos: Paris

DEMORGON, J. (2000). Complexité des cultures et de l'interculturel. Anthropos: Paris

DEMORGON, J. ET LIPIANSKY, M. (1999). Guide de l'interculturel en formation. Éditions Retz: Paris.

FARON, L. (1969). Los mapuches, su estructura social. Instituto Indigenista Interamericano, México.

FOERSTER, R.; MONTECINO, S. (1988). Organizaciones, Líderes y Contiendas Mapuches (1900-1970). Centro de la Mujer, Temuco.
FORNET-BETANCOURT R. (2001). "Filosofía e interculturalidad en América Latina, Intento de introducción no filosófica". En Heise M. (ed.), Interculturalidad. Creación de un concepto y desarrollo de una actitud. Lima: Inversiones Hathuey S.A.C., pp. 63-73.

GLAZER, N. (1996). "Multiculturalism and American Exceptionalisme", communication au colloque Multiculturalism, Minorities and Citizenship, Florence, avril 1996.

Gosselin, G. (1994). Les sociétés pluriculturelles. L'Harmattan: Paris.

HESS, R; WULF, C. (1999). Parcours, passages et paradoxes de l'interculturel, Ed. Anthropos: Paris.

MARROQUIN, A. (1977). Balance del Indigenismo. Ediciones Especiales: 76, Instituto Indigenista Interamericano, México.

MUÑOZ, J.; VELARDE, J. (2000). Compendio de epistemología. Editorial Trotta, Madrid.

ORELLANA, M. (1994). Prehistoria etnología de Chile. Santiago: Universidad de Chile, Bravo y Allende editores.

QUILAQUEO, D.; QUINTRIQUEO, S.; CÁRDENAS, P. (2005). Educación, curriculum e interculturalidad. Elementos sobre formación de profesores en contexto mapuche. Frasis editores, Santiago.

QUILAQUEO, D. (2005). "Educación intercultural desde la Teoría del Control Cultural en contexto de diversidad sociocultural mapuche". Cuadernos Interculturales, Año $3 \mathrm{~N}^{\circ} 4$. Centro de Estudios Interculturales y del Patrimonio (CEIP), Universidad de Valparaíso, pp. 37-50.

QUILAQUEO, D.; QUINTRIQUEO, S. (2008). "Formación docente en educación intercultural para contexto mapuche en Chile". Cuadernos Interculturales, primer semestre, año/ vol. 6, número 10, Universidad de Valparaíso, Viña del Mar, Chile, pp. 91-110

REICHENBACH, H. (1953). La filosofía científica. Fondo de Cultura Económica, MéxicoBuenos Aires.

SALAS, R. (2003). Ética Intercultural. (Re) Lecturas del pensamiento latinoamericano. Ediciones UCSH, Santiago. 
TODOROV, T. (1991). Nosotros y los otros. Siglo Veintiuno Editores, S. A., México.

TODOROV, T. (1993). Las morales de la historia. Barcelona, España, Ediciones Paidós Ibérica, S. A.

VILLALOBOS, S.; ALDUNATE, C.; ZAPATER, H.; MÉNDEZ, L. Y BASCUÑAN, C. (1982). Relaciones fronterizas en la Araucanía. Ediciones Universidad Católica de Chile, Santiago.

WIEVIORKA, M. (1996). Une société fragmentée? Le multiculturalisme en débat. La Découverte, Paris. 